Published in final edited form as:

Curr Cardiovasc Imaging Rep. 2018 June ; 11(6): . doi:10.1007/s12410-018-9452-6.

\title{
Molecular Imaging of Inflammation in Ischemic Heart Disease
}

\author{
Isaac Bakerman, BS ${ }^{1}$, Mirwais Wardak, PhD $^{2,3,4}$, and Patricia K. Nguyen, MD ${ }^{1,4,5}$ \\ ${ }^{1}$ Department of Medicine, Division of Cardiovascular Medicine, Stanford University, 300 Pasteur \\ Drive, Stanford University, CA 94305 \\ 2Department of Radiology, 300 Pasteur Drive, Stanford University, CA 94305 \\ ${ }^{3}$ Molecular Imaging Program at Stanford (MIPS), 300 Pasteur Drive, Stanford University, CA \\ 94305 \\ ${ }^{4}$ Stanford Cardiovascular Institute, Stanford University, 265 Campus Drive, Stanford, CA 94305 \\ ${ }^{5}$ Cardiology Section, Veterans Affairs Palo Alto Health Care Administration, 3801 Miranda Ave, \\ Palo Alto, CA 94304
}

\begin{abstract}
Purpose of Review-Ischemic heart disease is caused by atherosclerosis, the build-up of plaque in the coronary arteries, which can lead to the development of heart attacks and heart muscle damage. Despite the advent of medical and surgical therapy to prevent and treat atherosclerosis and its adverse clinical effects, ischemic heart disease remains a leading cause of morbidity and mortality. Recent studies have suggested that the immune system may play a greater role in the development of plaque rupture and adverse left ventricular remodeling after myocardial infarction. Understanding the molecular processes by which inflammation contributes to the pathophysiology of ischemic heart disease is, therefore, worthwhile. This review focuses on new molecular imaging techniques to visualize immune cells to study their contribution to ischemic heart disease.
\end{abstract}

Recent Findings-A common technique applied to imaging inflammation in ischemic heart disease is targeting the up-regulation and trafficking of immune cells, which may contribute to the adverse consequences associated with atherosclerosis. In the past five years, advances in cell labeling for imaging with PET and MRI, including radioisotopes and nanoparticles, have confirmed that inflammatory cells can be visualized in vivo and in greater abundance in unstable cardiovascular disease and in areas of ischemic damage. The major criticisms of these studies to date include their small sample size, lack of histological correlation, limited association with longterm outcomes, and bias toward macrophage imaging.

Summary-While much progress has been made in imaging inflammation in ischemic heart disease over the past five years, additional studies in larger cohorts with histological validation and outcome correlation are needed. Nevertheless, imaging inflammation using PET or MRI has the

\footnotetext{
Corresponding author: Patricia Nguyen, MD, Cardiology Section, Building 100, 3801 Miranda Ave, Palo Alto, CA 94304. Conflict of Interest All authors declare that they have no conflict of interest.

Human and Animal Rights and Informed Consent

This article does not contain any studies with human or animal subjects performed by any of the authors.
} 
potential to become an important adjunct tool to improve the diagnosis, risk stratification, and therapeutic monitoring of patients with ischemic heart disease.

\section{Keywords}

inflammation; ischemic heart disease; molecular imaging; atherosclerosis; positron emission tomography; nanoparticles

\section{Introduction}

Coronary heart disease (CHD) is one of the major causes of death worldwide. ${ }^{1}$ Patients with CHD have an increased risk for the development of acute coronary syndrome. Acute coronary syndrome (ACS) describes patients who present with 1) unstable angina, 2) near or complete occlusion of their coronary artery, or 3) myocardial infarction. The underlying pathologic mechanism of most ACS is atherosclerotic plaque rupture, ${ }^{1-5}$ that may be partly caused by inflammation within the plaque., ${ }^{2,3}$ Specifically, inflammatory cells including macrophages and $\mathrm{T}$ cells release factors that destabilize the fibrous cap covering the plaque, releasing its contents into the arterial lumen. ${ }^{2}$ When plaques rupture, their components block blood supply to the heart, resulting in acute myocardial injury. Innate and adaptive immune cells then migrate into the injured myocardium to initiate repair. Although inflammation is necessary for wound healing after injury, a deregulated immune response can lead to further insult. In fact, an excessive immune response has been associated with adverse remodeling, ${ }^{6}$ a maladaptive process that results in progressive left ventricular (LV) dilation to compensate for LV dysfunction and leads to the development of heart failure. ${ }^{7}$

Molecular Imaging provides a sensitive and Informative means to Identify, study, and diagnose the biological nature of atherosclerosis and monitor Its evolution. ${ }^{7-9}$ Because molecular Imaging can directly visualize Immune cells, It may be particularly effective at determining how inflammation contributes to the development of ischemic heart disease (IHD). Today clinical imaging strategies (e.g., x-ray coronary angiography, multi-slice computed tomography (CT), magnetic resonance imaging (MRI), and ultrasound) focus on identifying obstructive plaques that may subsequently lead to myocardial infarction and heart failure. None of these modalities, however, gives a direct non-invasive readout of inflammation, an important component of the vulnerable plaque. ${ }^{4}$ In light of the recent trial published in the New England Journal of Medicine, which showed reduction in major adverse cardiac events in patients treated with the anti-inflammatory agent canakinumab, ${ }^{10}$ there exists a critical need to explore whether molecular imaging techniques can help identify high-risk patients and improve their outcomes. Without these strategies, these patients may otherwise go undetected using conventional methods and may not derive benefit from anti-inflammatory therapies.

In this review, we will summarize recent strategies to image inflammation in the atherosclerotic plaque and the ischemic myocardium at the molecular level and discuss recent studies evaluating the feasibility of applying these techniques clinically. 


\section{Targeting Immune Cells Using Radioactive Isotopes and Nanoparticles}

A common technique applied to imaging inflammation in IHD is targeting the up-regulation and trafficking of cells in immune system. In the past, this has been achieved with labeling immune cells with radioactive isotopes for single photon emission computed tomography

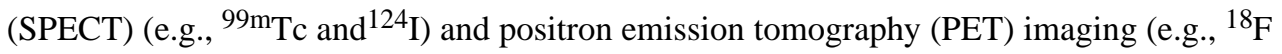
and ${ }^{64} \mathrm{Cu}$ ). More recently, nanomaterials have been used for visualization of immune cells. Although commonly used in fluorescence imaging, nano-based agents for MRI and PET have recently been described. ${ }^{11}$ The main advantage of nanomaterials over radiotracers is that they enable the attachment of imaging specific probes, targeting ligands, and therapeutic payloads in a single vector. Regulatory hurdles for the use of nanoparticles in humans remain a critical disadvantage.

Probably the most common nanomaterials for imaging immune cells are fluorescence-based and enable imaging with various modalities including microscopy, flow cytometry, endoscopy, optical tomography, and intra operative imaging. Fluorescent-based nanomaterials with high affinity to immune cells are either 1) fluorochrome labeled dextran particles, polyethylene glycol (PEG), silica nanoparticles, and other scaffolds or 2) quantum dots or up-converting nanoparticles (e.g., up-conversion occurs when two or more incident photons with low energy are absorbed and converted into a higher energy photon).

Fluorescence-based nanomaterials are the cornerstone of molecular imaging and have been used to validate the accuracy of other imaging agents. However, they remain limited in their clinical utility by the poor depth of penetration of fluorescence signals.

MRI visualization of inflammation requires accumulation of nanomaterials within immune cells. This results in shortening of $\mathrm{T} 2$ and $\mathrm{T} 2 *$ times of surrounding tissue causing a signal reduction (e.g., negative contrast) on MR images. Because areas of negative contrast appear as darkened areas, this signal is difficult to distinguish from internal bleeding, air-tissue boundaries, areas of high blood flow, and other susceptibility artifacts. As a result, there may be inaccurate identification and localization of inflammation using this technique. These limitations have led to the development of a number of positive contrast agents including extremely small supramagnetic iron oxide particles with a zwitterion coating that have high $\mathrm{T} 1$ constrast, ${ }^{12}$ nonspecific and specific targeted gadolinium positive contrast agents, ${ }^{13,14}$ and manganese contrast agents. ${ }^{15}$

Nanomaterial-based PET agents have increasingly been used in recent years. PET enables the quantification of positron labeled nanomaterials in organs and tissues. Most nanomaterials used for PET imaging include modified dextrans, graft-copolymers, and other scaffolds. In addition to ${ }^{18} \mathrm{~F}$ and ${ }^{64} \mathrm{Cu}$, the most common labeling radioisotopes include ${ }^{89} \mathrm{Zr}$, ${ }^{124} \mathrm{I}$, and ${ }^{76} \mathrm{Br}$. The choice of the radiolabeling agent depends on the circulating time of the nanomaterial with those with the longest circulating time requiring positron emitters with the longest half-life.

For reasons discussed below, monocytes/macrophages and lymphocytes are the most common targets for these probes. Thus, while other immune subsets may also play an important pathological role, this review will focus on how molecular imaging has 
interrogated the contribution of monocytes/macrophages and lymphocytes to the development and progression of IHD.

\section{Imaging Macrophages and Monocytes in the Atherosclerotic Plaque and Infarcted Myocardium}

Macrophages are tissue resident components of the innate and adaptive immune system. Monocytes are circulating cells in the blood stream that can differentiate into macrophages when they enter tissue. Both macrophages and monocytes perform various functions in host defense including antigen presentation and endocytosis (e.g., phagocytosis or pinocytosis). Because these cells can engulf foreign material via endocytosis, they can take up radiolabels or nanomaterials, enabling their in vivo visualization. These probes are taken up by both macrophages and monocytes in healthy and diseased tissues. The densities of these cells, however, are higher in diseased and inflamed tissue, which facilitates the interrogation of the relationship between inflammation and IHD with molecular imaging.

Although it remains unclear whether probe uptake occurs directly in tissue macrophages or, initially in circulating monocytes that then travel to the diseased tissue, previous studies support the latter scenario with respect to the infarcted myocardium. ${ }^{16}$ Leuschner et al, ${ }^{16}$ for example, studied the tissue kinetics and clearance of monocytes and macrophages in a murine model of IHD. In this study, they transplanted an infarcted heart from a mouse with an allelic variant of $C D 45$, a surface marker of blood cells, to a mouse with a different allelic variant of CD45. Using flow cytometric analysis of digested infarcted tissue, the authors followed the dynamic changes in the monocyte population at the site of the infarct at different time-points. The authors found an extremely short residence time for macrophages and sustained need for new macrophages replenished by splenic monocytopoeisis, suggesting that probes are taken by monocytes that differentiate into macrophages after they enter the tissue. Consistent with these findings, Montet-Abou, et al ${ }^{17}$ found that monocytes labeled with fluorescent iron particles migrated into the infarcted myocardium in a rat injury model on serial MRI and optical imaging.

Whether molecular imaging can distinguish between activated macrophages (e.g., those responding to cytokines released from other inflammatory cells and the tissue microenvironment) vs. resting macrophages (e.g., those that function in absence of inflammation) remains under investigation. Activated rather than resting macrophages drive the chronic inflammatory response seen in IHD. These cells release cytokines, chemokines, digestive enzymes, prostaglandins, and reactive oxygen species that contribute to atherosclerotic plaque progression and instability as well as adverse left ventricular remodeling. Although activated macrophages can be further divided into the pathological M1 vs. the reparative M2 phenotype based on stimuli in the microenvironment, intermediate phenotypes have been described in vivo. ${ }^{18}$ Both M1 and M2 subtypes have been efficiently labeled in vivo. In order for molecular imaging to distinguish between these different physiological states, however, it will be important to identify specific surface cell markers that can be targeted by imaging probes. For example, it was found that classically activated macrophages express a receptor for folic acid (e.g., folate receptor- $\beta$ ), which is absent on resting macrophages. ${ }^{19}$ As a result, imaging agents targeting folate receptor- $\beta$ agents have 
been developed. In previous studies, these agents have successfully imaged activated macrophages accumulating in atherosclerotic plaques of mice that were fed a high fat diet using gamma scintigraphy ${ }^{20}$ and in carotid plaques explanted from patients undergoing carotid endarterectomy using fluorescence imaging. ${ }^{21}$ More recently, in a pilot study, Jager, et $\mathrm{al}^{22}$ found that ${ }^{99 \mathrm{~m}} \mathrm{Tc}-\mathrm{EC} 20$, which targets folate receptor- $\beta$, could potentially differentiate between M1 and M2 subtypes. Specifically, ${ }^{99 \mathrm{~m}} \mathrm{Tc}-\mathrm{EC} 20$ preferentially labeled macrophages expressing both folate receptor- $\beta$ and Cd163, which are M2 macrophage surface markers (Figure 1). Because M2 subtypes release more digestive proteins that can contribute to plaque vulnerability, the authors suggest that ${ }^{99 \mathrm{~m}} \mathrm{Tc}-\mathrm{EC} 20$ can potentially identify vulnerable plaque. Its potential clinical utility, however, requires further investigation.

\section{Imaging Lymphocytes in IHD}

Although monocytes/macrophages have received the majority of focus in previous studies, increasing evidence suggests that the adaptive immune system enhances and regulates inflammation in IHD. Both T and B cells have been found in the atherosclerotic plaque, with the former representing the second largest leukocyte population in the plaque after monocytes. The specific antigen(s) that activate the adaptive immune response in IHD, however, have not been identified although most studies have suggested that oxidized lowdensity lipoprotein may be one of the likely culprits. ${ }^{23}$

Unlike monocytes/macrophages, lymphocytes have limited uptake of probes via endocytosis. To enable lymphocyte imaging, lymphocytes can be directly labeled ex vivo ${ }^{24}$ or genetically modified to express a reporter gene ex vivo. ${ }^{25}$ Although metabolic probes such as ${ }^{18}$ F-FDG and ${ }^{18}$ F-FLT can be used for in vivo imaging, ${ }^{26}$ they are not specific for lymphocytes and target any proliferating cell. Improved imaging specificity can be achieved by using radiolabeled monoclonal antibodies that bind lymphocyte specific markers such as $\mathrm{CD} 25, \mathrm{CD} 2, \mathrm{CD} 5, \mathrm{CD} 3^{27}$ and $\mathrm{T}$ cell receptor. ${ }^{28}$ Further characterization of $\mathrm{T}$ cell subsets using anti-CD4 and anti-CD8 conjugated to PET isotopes (e.g., ${ }^{64} \mathrm{Cu}$ and exotic radionuclides like ${ }^{89} \mathrm{Zr}$ ) was recently achieved by Tavaré, et al, ${ }^{29,30}$ who successfully demonstrated visualization of CD4 and CD8 T cells in the spleen and lymph nodes of wild type mice. Although monoclonal antibodies have been used for imaging lymphocytes post hematopoietic transplant (e.g., ${ }^{89} \mathrm{Zr}$ conjugated to anti-CD8 and anti-CD4), ${ }^{30}$ acute airway delayed sensitivity (e.g., $\mathrm{Cu}^{64}$ conjugated to anti-T cell receptor), ${ }^{31}$ and tumors in mice (e.g., ${ }^{89} \mathrm{Zr}$ conjugated to the $\mathrm{T}$ cell receptor), ${ }^{28}$ it has yet to be applied in evaluating IHD. Identification of unique surface markers will be needed to further refine imaging for $\mathrm{T}$ cell subsets (e.g., memory $\mathrm{T}$ cells, regulator T cells) in IHD.

To achieve good target/background ratio, however, images generated by targeting lymphocytes with monoclonal antibodies must be delayed for 6-24 hours, which may negatively impact the throughput of this strategy if applied clinically. The technique, however, has minimal side effects. Of note, the only significant side effect after administration of monoclonal antibodies is the induction of human murine antibodies, which is a host response to foreign antigens that can affect the probe's bio-distribution, image quality, and interpretation findings clinically if the probe is administered repeatedly. 


\section{Inflammation Imaging in Patients with IHD}

Evaluation of inflammation in IHD is not routinely performed in the clinical setting and remains under investigation. Over the last five years, there appears to be more clinical studies evaluating inflammation in the atherosclerotic plaque than in the ischemic myocardium (Table 1). In the past, the majority of clinical studies have focused on the use of ${ }^{18} \mathrm{~F}-\mathrm{FDG}$ although it remains a nonspecific marker of inflammation. Given the limitations of ${ }^{18}$ F-FDG as outlined below, more recent clinical imaging studies have primarily targeted surface markers primarily on macrophages. Below is a selected discussion of recent clinical imaging studies evaluating inflammation in the atherosclerotic plaque and ischemic myocardium.

\section{Imaging atherosclerotic plaque in patients with IHD}

FDG PET is the most well studied technique for imaging plaque inflammation. In several prospective studies, ${ }^{32,33}$ the utility of this approach has been successfully demonstrated in imaging plaque in vivo in the carotid, vertebral, iliac, femoral, and coronary arteries in symptomatic patients. Importantly, higher levels of FDG uptake on baseline scans had poorer outcomes, including a higher incidence of recurrent stroke, death and revascularization. While results from these early studies are promising, more conclusive evidence of the utility of FDG is needed and may soon come from the BioImaging study, ${ }^{34} \mathrm{a}$ prospective study that enrolled 6104 patients at risk for coronary artery disease. These patients will undergo imaging studies including vascular ultrasound, MRI, CT, and FDG PET/CT and are then followed for three years for clinical events. Despite its reported utility, however, the major limitation of FDG remains its nonspecific uptake by any cell with high metabolic activity, resulting in high myocardial background activity, inability to distinguish inflammatory subtypes (e.g., macrophages vs. T cells), and uptake in other metabolically active cells (e.g., endothelial cells). In addition, FDG uptake can be influenced by mechanisms other than inflammation such as hypoxia, ${ }^{35}$ increasing the likelihood of false positives. Finally, avid uptake of FDG by distressed myocytes reliant on glycolysis remains a critical limitation of FDG imaging of coronary atherosclerosis despite reports that background myocyte signal can be somewhat attenuated by the ingestion of meals low in carbohydrates the day before the scan. ${ }^{5}$

Alternative radiotracers under investigation that have higher specificity than FDG include tracers that specifically target surface markers. While clinical studies targeting lymphocyte specific markers are nonexistent to date, a number of studies have investigated the utility of imaging surface markers on macrophages as a strategy to increase imaging specificity. ${ }^{11} \mathrm{C}$ PK11195 $\left({ }^{11} \mathrm{C}\right.$ - $N$-methyl- $N$-[1-methylpropyl]-1-[2-chlorophenyl]-isoquin-oline-3 carboxamide) and ${ }^{18}$ F-GE-180 ( $S$ - $N, N$-diethyl-9-[2- ${ }^{18}$ F-fluoroethyl]-5-methoxy 2,3,4,9tetrahydro-1H-carbamazole-4-carboxamide), for example, target translocator proteins (TSPOs) expressed on activated macrophages. In a preliminary study, a higher uptake of ${ }^{11} \mathrm{C}-\mathrm{PK} 11195$ was found in symptomatic versus asymptomatic carotid arteries in vivo. The signal co-localized with activated macrophages detected on autoradiography and CD68 staining of ex vivo histology. ${ }^{36}$ The utility of this tracer for imaging vessel inflammation, however, is limited by its widespread uptake in the healthy vessel wall. ${ }^{18} \mathrm{~F}-\mathrm{GE}-180$ is a newer generation of TSPO tracer that offers a longer half-life and higher binding capacity 
due to the use of ${ }^{18} \mathrm{~F}$ as its positron emitter and replacement of PK11195 with GE-180 as a ligand, respectively. Although ${ }^{8} \mathrm{~F}-\mathrm{GE}-180$ imaging has resulted in improved signal-to-noise ratio and lower nonspecific binding in animal models of cerebral infarction, it has yet been applied to image inflammation associated with IHD. ${ }^{37}$ The use of both ${ }^{11} \mathrm{C}-\mathrm{PK} 11195$ and ${ }^{18} \mathrm{~F}-\mathrm{GE}-180$ for imaging inflammation, however, may be limited by the presence of genetic polymorphisms that affect the binding capacity of these agents in some individuals ${ }^{38}$ as well as high myocardial uptake making coronary plaque imaging challenging.

Other surface receptors on macrophages have been utilized for imaging atherosclerosis including folate receptor- $\beta$ (as discussed previously), ${ }^{22}$ lectin-like oxidized LDL receptor (LOX)- $1,{ }^{39}$ mannose receptor, ${ }^{40}$ and somatostatin (SST) receptors. Of these, only the radiotracer DOTATATE ([1,4,7,10-tetraazacyclododecane-N, N', N", N"'-tetraacetic acid]-dPhe1, Tyr3-octrotate), which targets SST receptors, has been evaluated in humans in vivo. In a preliminary study of 44 asymptomatic patients, ${ }^{41}{ }^{68} \mathrm{Ga}$-DOTATATE uptake was higher in fibrotic plaques compared to normal coronary arteries. Importantly, a recent study compared detection of atherosclerotic inflammation by ${ }^{68}$ Ga-DOTATATE with ${ }^{18}$ F-FDG PET imaging in 42 patients, 18 patients with stable cardiovascular disease (e.g., stable angina or asymptomatic atherosclerosis) and 24 patients with unstable cardiovascular disease (e.g., acute coronary syndrome or TIA/stroke, with a subset of 8 undergoing carotid endarterectomy). ${ }^{42}$ The study confirmed that ${ }^{68} \mathrm{Ga}$-DOTATATE binds to macrophages in excised carotid specimens based on gene expression analysis, correctly identifies culprit vs. nonculprit lesions in patients with unstable disease. ${ }^{68}$ Ga-DOTATATE uptake also correlated with high risk plaque features determined by CT. Coronary ${ }^{68} \mathrm{Ga}$-DOTATATE was interpretable in all patients unlike ${ }^{18}$ F-FDG PET, which could not be interpreted in 27 (64\%) patients due to high myocardial background signal. These findings are in direct contrast to the negative study reported by Wan, et al. ${ }^{43}$ showing that ${ }^{68}$ Ga-DOTATATE activity did not differ between symptomatic and contralateral plaques from patients who suffered transient ischemic attacks and were scheduled for carotid endarterectomy. The authors suggest that the lack of difference may be due to the imaging characteristics of ${ }^{68} \mathrm{Ga}$-DOTATATE, which may be less sensitive to subtle differences in uptake than other tracers like ${ }^{64} \mathrm{Cu}$ DOTATATE. ${ }^{44}$ Supporting this hypothesis, a head-to-head comparison of these two tracers in 60 patients showed that uptake of ${ }^{64} \mathrm{Cu}$-DOTATATE but not ${ }^{68} \mathrm{Ga}$-DOTATATE in large arterial segments was more readily identified and correlated with cardiac risk factors including body mass index, smoking, diabetes and coronary artery calcium score (Figure 2). ${ }^{45}$ While these results are promising, large human trials with histological validation are needed to further validate the use of these tracers to image plaque inflammation.

Plaque visualization can also be accomplished using nanoparticles that rely on the phagocytic abilities of macrophages to take up imaging agents. Although uptake is nonspecific, nanoparticles have specialized optical, magnetic and chemical properties that provide better contrast enhancement, increased sensitivity, improved bio-distribution, and higher spatial and temporal resolution. In magnetic resonance imaging, for example, studies have found that injected superparamagnetic iron oxide particles (SPIONs) engulfed by macrophages at the site of inflammation are more prominent in rupture prone compared to stable plaques ${ }^{46}$ the quantity of SPION particles in plaques decreased with atorvastatin treatment, ${ }^{47}$ and the amount of SPION uptake remained unchanged over time in stable, 
asymptomatic plaques. ${ }^{48}$ Taken together, these findings suggest that SPIONs may be used to identify vulnerable plaques, monitor disease progression over time, and assess therapeutic response. Larger, multi-center trials combined with histological validation and outcome correlation are needed before widespread clinical application of SPIONs for the management of atherosclerosis can be recommended. Of note, the FDA recently approved a first-in-human trial of a nanoparticle PET radiotracer targeting natriuretic peptide receptor $\mathrm{C}$ (NPR-C), which is differentially expressed in endothelial cells, smooth muscle cells and macrophages within the plaque of $\mathrm{APOE}^{-/-}$deficient mice. ${ }^{49}$ In this trial, patients scheduled for carotid endarterectomy and enrolled in this single center, open label imaging study will undergo $\mathrm{Cu}$ [64]-25\%-CANF-Comb PET-MR imaging of both carotid arteries to determine the feasibility of this tracer to assess disease severity. The presence of NPR-C will be confirmed by RT-PCR of excised carotid endarterectomy specimens.

\section{Imaging the ischemic myocardium}

The techniques described above have also been applied for imaging inflammation in the myocardium after ischemic injury. Within 30 minutes of acute myocardial injury, neutrophils and pro inflammatory monocytes infiltrate the damaged tissue.$^{50}$ Four days post-infarction, a less inflammatory phase develops which is characterized by recruitment of Ly6Clow macrophages whose appearance is regulated by T- and B-lymphocytes. ${ }^{51-53}$ If there is an overzealous influx of pro-inflammatory cells or delay in the transition to the less inflammatory phase, infarct healing is compromised and heart failure develops. ${ }^{53,54}$ Taken together, these findings suggest that imaging of immune subsets in the ischemic myocardium can improve risk stratification and identify potential immune modulating therapies to halt the development of heart failure.

While ${ }^{18}$ F-FDG may be feasible to image inflammation in mice after ischemic injury, ${ }^{55}$ it remains challenging in humans. Unlike mice, inflamed ischemic tissue in humans is often interspersed with normal surviving myocytes, creating a heterogeneous distribution that can confound clinical interpretation. To explore whether ${ }^{18} \mathrm{~F}-\mathrm{FDG}$ uptake can be used clinically post MI, Rischpler, et al. ${ }^{56}$ prospectively evaluated 49 patients with STEMI using ${ }^{18}$ F-FDG PET/MRI 5 days after PCI with a follow-up cardiac MRI after 6-9 months. The mean standardized uptake value (SUV mean) of ${ }^{18}$ F-FDG was detected in transmural scars and was associated with left ventricular dysfunction independent of infarct size measured by late gadolinium enhancement. Unlike the extent of delayed enhancement and SUV mean of ${ }^{18} \mathrm{~F}$ FDG, the extent of FDG uptake did not correlate significantly with outcome. This likely occurred because the detected signal not only represents inflammatory cells, but also indicates the presence of compromised but viable myocytes. These findings highlight the need for imaging strategies specifically targeting inflammation.

In addition to FDG PET imaging, a handful of studies have targeted macrophages for visualizing inflammation in the ischemic myocardium. The feasibility of ferumoxytolenhanced MRI was demonstrated in sequential exams of 16 patients after acute myocardial infarction. ${ }^{57}$. Ultra small SPIONs were detected in the infarct and, to a lower extent, the remote area with increasing uptake at 48 hours compared to baseline. Inflammation appears to peak at 2-3 days and dissipate by week 2, as suggested by a second study of 31 patients 
undergoing serial ferumoxytol-enhanced MRI. ${ }^{58}$ The presence of macrophages engulfing SPIONs in the infarct zone was confirmed by histology. Additional studies that include histological confirmation and outcome correlation (e.g., changes in left ventricular function, development of heart failure, death) are needed to determine whether in vivo monitoring of inflammation and its modulation will be clinically valuable.

\section{Conclusions}

Although recent studies IHD including acute coronary syndrome and heart failure, there are limited therapies for IHD directed at modulating inflammation. Molecular imaging may help address this gap by enabling the visualization of inflammatory cells in vivo and determining their contribution to disease development. While clinical studies using nonspecific and specific imaging agents have shown increased uptake of these agents in unstable plaques as well as in infarcted tissue, these studies have small sample sizes and often lack histological confirmation and correlation to long-term outcomes. Future studies are needed to address these shortcomings before inflammation imaging can play an important role in the management of IHD.

\section{Acknowledgment}

We would like to thank the National Institutes of Health grants HL 134830-01 (PKN) for funding support.

\section{Abbreviations}

ACS:

CHD:

11C-PK11195:

CT:

DOTATATE:

FDG:

${ }^{18}$ F-GE-180:

IHD:

LOX-1:

LV:

MRI:

NPR-C:

PEG: acute coronary syndrome

coronary heart disease

${ }^{11} \mathrm{C}-\mathrm{N}$-methyl- $N$-[1-methylpropyl]-1-[2-chlorophenyl]isoquinoline-3 car-box-amide)

computed tomography

[1,4,7,10-tetraazacyclododecane-N, N', N", N"'-tetraacetic acid]-d-Phe1, Tyr3-octrotate

$\left[{ }^{18}\right.$ F]fluorodeoxyglucose

$S$ - $N, N$-diethyl-9-[2- ${ }^{18}$ F-fluoroethyl]-5-methoxy 2,3,4,9tetrahydro-1H-carbamazole-4-carboxamide

ischemic heart disease

lectin-like oxidized LDL receptor-1

left ventricular

magnetic resonance imaging

natriuretic peptide receptor $\mathrm{C}$

polyethylene glycol 


$\begin{array}{ll}\text { PET: } & \text { positron emission tomography } \\ \text { SPECT: } & \text { single photon emission computed tomography } \\ \text { SPIONs: } & \text { superparamagnetic iron oxide particles } \\ \text { SST: } & \text { somatostatin } \\ \text { SUVmean: } & \text { mean standardized uptake value } \\ \text { TSPOs: } & \text { translocator proteins }\end{array}$

\section{References}

Papers of particular interest, published recently, have been highlighted as:

* Of importance

** Of major importance

1. Benjamin EJ, Blaha MJ, Chiuve SE, et al. Heart disease and stroke statistics-2017 update: a report from the American Heart Association. Circulation. 2017;135(10):e146-e603. [PubMed: 28122885]

2. Hansson GK. Inflammation, atherosclerosis, and coronary artery disease. The New England Journal of Medicine. 2005;352(16):1685-1695. [PubMed: 15843671]

3. Hiari N, Rudd JH. FDG PET imaging and cardiovascular inflammation. Current Cardiology Reports. 2011;13(1):43-48. [PubMed: 20949379]

4. Rudd JH, Warburton EA, Fryer TD, et al. Imaging atherosclerotic plaque inflammation with [18F]fluorodeoxyglucose positron emission tomography. Circulation. 2002;105(23):2708-2711. [PubMed: 12057982]

5. Tarkin JM, Joshi FR, Rudd JH. PET imaging of inflammation in atherosclerosis. Nature Reviews. Cardiology. 2014;11(8):443-457. [PubMed: 24913061]

6. Westman PC, Lipinski MJ, Luger D, et al. Inflammation as a driver of adverse left ventricular remodeling after acute myocardial infarction. Journal of the American College of Cardiology. 2016;67(17):2050-2060. [PubMed: 27126533]

7. Sutton MG, Sharpe N. Left ventricular remodeling after myocardial infarction: pathophysiology and therapy. Circulation. 2000;101(25):2981-2988. [PubMed: 10869273]

8. Phelps ME. Positron emission tomography provides molecular imaging of biological processes. Proceedings of the National Academy of Sciences of the United States of America. 2000;97(16): 9226-9233. [PubMed: 10922074]

9. Phelps ME. PET: the merging of biology and imaging into molecular imaging. Journal of Nuclear Medicine. 2000;41(4):661-681. [PubMed: 10768568]

10. Ridker PM, Everett BM, Thuren T, et al. Antiinflammatory therapy with canakinumab for atherosclerotic disease. The New England Journal of Medicine. 2017;377(12):1119-1131. [PubMed: 28845751]

11. Weissleder R, Nahrendorf M, Pittet MJ. Imaging macrophages with nanoparticles. Nature Materials. 2014;13(2):125-138. [PubMed: 24452356]

12. Wei H, Bruns OT, Kaul MG, et al. Exceedingly small iron oxide nanoparticles as positive MRI contrast agents. Proceedings of the National Academy of Sciences of the United States of America. 2017;114(9):2325-2330. [PubMed: 28193901]

13. Calcagno C, Ramachandran S, Millon A, Robson PM, Mani V, Fayad Z. Gadolinium-based contrast agents for vessel wall magnetic resonance imaging (MRI) of atherosclerosis. Current Cardiovascular Imaging Reports. 2013;6(1):11-24. [PubMed: 23539505]

14. Marangoni VS, Neumann O, Henderson L, et al. Enhancing T1 magnetic resonance imaging contrast with internalized gadolinium(III) in a multilayer nanoparticle. Proceedings of the National 
Academy of Sciences of the United States of America. 2017;114(27):6960-6965. [PubMed: 28630340]

15. Huang G, Li H, Chen J, et al. Tunable T1 and T2 contrast abilities of manganese-engineered iron oxide nanoparticles through size control. Nanoscale. 2014;6(17):10404-10412. [PubMed: 25079966]

16. Leuschner F, Rauch PJ, Ueno T, et al. Rapid monocyte kinetics in acute myocardial infarction are sustained by extramedullary monocytopoiesis. The Journal of Experimental Medicine. 2012;209(1):123-137. [PubMed: 22213805]

17. Montet-Abou K, Daire JL, Hyacinthe JN, et al. In vivo labelling of resting monocytes in the reticuloendothelial system with fluorescent iron oxide nanoparticles prior to injury reveals that they are mobilized to infarcted myocardium. European Heart Journal. 2010;31(11):1410-1420. [PubMed: 20023288]

18. Sica A, Mantovani A. Macrophage plasticity and polarization: in vivo veritas. The Journal of Clinical Investigation. 2012;122(3):787-795. [PubMed: 22378047]

19. Xia W, Hilgenbrink AR, Matteson EL, Lockwood MB, Cheng JX, Low PS. A functional folate receptor is induced during macrophage activation and can be used to target drugs to activated macrophages. Blood. 2009;113(2):438-446. [PubMed: 18952896]

20. Ayala-Lopez W, Xia W, Varghese B, Low PS. Imaging of atherosclerosis in apoliprotein e knockout mice: targeting of a folate-conjugated radiopharmaceutical to activated macrophages. Journal of Nuclear Medicine. 2010;51(5):768-774. [PubMed: 20395331]

21. Jager NA, Westra J, van Dam GM, et al. Targeted folate receptor beta fluorescence imaging as a measure of inflammation to estimate vulnerability within human atherosclerotic carotid plaque. Journal of Nuclear Medicine. 2012;53(8):1222-1229. [PubMed: 22855837]

22. Jager NA, Westra J, Golestani R, et al. Folate receptor-beta imaging using 99mTc-folate to explore distribution of polarized macrophage populations in human atherosclerotic plaque. Journal of Nuclear Medicine. 2014;55(12):1945-1951. [PubMed: 25359878]

23. Shah PK, Chyu KY, Dimayuga PC, Nilsson J. Vaccine for atherosclerosis. Journal of the American College of Cardiology. 2014;64(25):2779-2791. [PubMed: 25541132]

24. Griessinger CM, Kehlbach R, Bukala D, et al. In vivo tracking of Th1 cells by PET reveals quantitative and temporal distribution and specific homing in lymphatic tissue. Journal of Nuclear Medicine. 2014;55(2):301-307. [PubMed: 24434289]

25. Koya RC, Mok S, Comin-Anduix B, et al. Kinetic phases of distribution and tumor targeting by $\mathrm{T}$ cell receptor engineered lymphocytes inducing robust antitumor responses. Proceedings of the National Academy of Sciences of the United States of America. 2010;107(32):14286-14291. [PubMed: 20624956]

26. Aarntzen EH, Srinivas M, De Wilt JH, et al. Early identification of antigen-specific immune responses in vivo by [18F]-labeled 3'-fluoro-3'-deoxy-thymidine ([18F]FLT) PET imaging. Proceedings of the National Academy of Sciences of the United States of America. 2011;108(45): 18396-18399. [PubMed: 22025695]

27. Malviya G, D'Alessandria C, Bonanno E, et al. Radiolabeled humanized anti-CD3 monoclonal antibody visilizumab for imaging human T-lymphocytes. Journal of Nuclear Medicine. 2009;50(10):1683-1691. [PubMed: 19759100]

28. Mall S, Yusufi N, Wagner R, et al. Immuno-PET imaging of engineered human T cells in tumors. Cancer Research. 2016;76(14):4113-4123. [PubMed: 27354381]

29. Tavare R, McCracken MN, Zettlitz KA, et al. Engineered antibody fragments for immuno-PET imaging of endogenous CD8+ T cells in vivo. Proceedings of the National Academy of Sciences of the United States of America. 2014;111(3):1108-1113. [PubMed: 24390540]

30. Tavare R, McCracken MN, Zettlitz KA, et al. Immuno-PET of murine T cell reconstitution postadoptive stem cell transplantation using anti-CD4 and anti-CD8 cys-diabodies. Journal of Nuclear Medicine. 2015;56(8):1258-1264. [PubMed: 25952734]

31. Griessinger CM, Maurer A, Kesenheimer C, et al. 64Cu antibody-targeting of the T-cell receptor and subsequent internalization enables in vivo tracking of lymphocytes by PET. Proceedings of the National Academy of Sciences of the United States of America. 2015;112(4):1161-1166.

[PubMed: 25587131] 
32. Menezes LJ, Kotze CW, Hutton BF, et al. Vascular inflammation imaging with 18F-FDG PET/CT: when to image? Journal of Nuclear Medicine. 2009;50(6):854-857. [PubMed: 19443587]

33. Rogers IS, Nasir K, Figueroa AL, et al. Feasibility of FDG imaging of the coronary arteries: comparison between acute coronary syndrome and stable angina. JACC. Cardiovascular Imaging. 2010;3(4):388-397. [PubMed: 20394901]

34. Baber U, Mehran R, Sartori S, et al. Prevalence, impact, and predictive value of detecting subclinical coronary and carotid atherosclerosis in asymptomatic adults: the BioImage study. Journal of the American College of Cardiology. 2015;65(11):1065-1074. [PubMed: 25790876]

35. Folco EJ, Sheikine Y, Rocha VZ, et al. Hypoxia but not inflammation augments glucose uptake in human macrophages: Implications for imaging atherosclerosis with 18fluorine-labeled 2-deoxy-Dglucose positron emission tomography. Journal of the American College of Cardiology. 2011;58(6):603-614. [PubMed: 21798423]

36. Bird JL, Izquierdo-Garcia D, Davies JR, et al. Evaluation of translocator protein quantification as a tool for characterising macrophage burden in human carotid atherosclerosis. Atherosclerosis. 2010;210(2):388-391. [PubMed: 20056222]

37. Boutin H, Murray K, Pradillo J, et al. 18F-GE-180: a novel TSPO radiotracer compared to 11C-RPK11195 in a preclinical model of stroke. European Journal of Nuclear Medicine and Molecular Imaging. 2015;42(3):503-511. [PubMed: 25351507]

38. Guo Q, Colasanti A, Owen DR, et al. Quantification of the specific translocator protein signal of 18F-PBR111 in healthy humans: a genetic polymorphism effect on in vivo binding. Journal of Nuclear Medicine. 2013;54(11):1915-1923. [PubMed: 24071511]

39. Li D, Patel AR, Klibanov AL, et al. Molecular imaging of atherosclerotic plaques targeted to oxidized LDL receptor LOX-1 by SPECT/CT and magnetic resonance. Circulation. Cardiovascular Imaging. 2010;3(4):464-472. [PubMed: 20442371]

40. Tahara N, Mukherjee J, de Haas HJ, et al. 2-deoxy-2-[18F]fluoro-D-mannose positron emission tomography imaging in atherosclerosis. Nature Medicine. 2014;20(2):215-219.

41. Mojtahedi A, Alavi A, Thamake S, et al. Assessment of vulnerable atherosclerotic and fibrotic plaques in coronary arteries using (68)Ga-DOTATATE PET/CT. American Journal of Nuclear Medicine and Molecular Imaging. 2015;5(1):65-71. [PubMed: 25625028]

42. Tarkin JM, Joshi FR, Evans NR, et al. Detection of atherosclerotic inflammation by (68)GaDOTATATE PET compared to [(18)F]FDG PET imaging. Journal of the American College of Cardiology. 2017;69(14):1774-1791. [PubMed: 28385306]

43. Wan MYS, Endozo R, Michopoulou S, et al. PET/CT Imaging of unstable carotid plaque with (68)Ga-labeled somatostatin receptor ligand. Journal of Nuclear Medicine. 2017;58(5):774-780. [PubMed: 27932558]

44. Pedersen SF, Sandholt BV, Keller SH, et al. 64Cu-DOTATATE PET/MRI for Detection of activated macrophages in carotid atherosclerotic plaques: studies in patients undergoing endarterectomy. Arteriosclerosis, Thrombosis, and Vascular Biology. 2015;35(7):1696-1703.

45. Malmberg C, Ripa RS, Johnbeck CB, et al. 64Cu-DOTATATE for Noninvasive assessment of atherosclerosis in large arteries and its correlation with risk factors: head-to-head comparison with 68Ga-DOTATOC in 60 patients. Journal of Nuclear Medicine. 2015;56(12):1895-1900. [PubMed: 26429961]

46. Howarth SP, Tang TY, Trivedi R, et al. Utility of USPIO-enhanced MR imaging to identify inflammation and the fibrous cap: a comparison of symptomatic and asymptomatic individuals. European Journal of Radiology. 2009;70(3):555-560. [PubMed: 18356000]

47. Tang TY, Howarth SP, Miller sR, et al. The ATHEROMA (Atorvastatin Therapy: Effects on Reduction of Macrophage Activity) Study. Evaluation using ultrasmall superparamagnetic iron oxide-enhanced magnetic resonance imaging in carotid disease. Journal of the American College of Cardiology. 2009;53(22):2039-2050. [PubMed: 19477353]

48. Sadat U, Howarth SP, Usman A, Tang TY, Graves MJ, Gillard JH. Sequential imaging of asymptomatic carotid atheroma using ultrasmall superparamagnetic iron oxide-enhanced magnetic resonance imaging: a feasibility study. Journal of Stroke and Cerebrovascular Diseases. 2013;22(8):e271-276. [PubMed: 22841932] 
49. Woodard PK, Liu Y, Pressly ED, et al. Design and modular construction of a polymeric nanoparticle for targeted atherosclerosis positron emission tomography imaging: A Story of $25 \%$ (64)Cu-CANF-Comb. Pharmaceutical Research. 2016;33(10):2400-2410. [PubMed: 27286872]

50. Jung K, Kim P, Leuschner F, et al. Endoscopic time-lapse imaging of immune cells in infarcted mouse hearts. Circulation Research. 2013; 112(6):891-899. [PubMed: 23392842]

51. Hilgendorf I, Swirski FK, Robbins CS. Monocyte fate in atherosclerosis. Arteriosclerosis, Thrombosis, and Vascular Biology. 2015;35(2):272-279.

52. Weirather J, Hofmann UD, Beyersdorf N, et al. Foxp3+ CD4+ T cells improve healing after myocardial infarction by modulating monocyte/macrophage differentiation. Circulation Research. 2014; 115(1):55-67. [PubMed: 24786398]

53. Zouggari Y, Ait-Oufella H, Bonnin P, et al. B lymphocytes trigger monocyte mobilization and impair heart function after acute myocardial infarction. Nature Medicine. 2013; 19(10): 12731280.

54. Panizzi P, Swirski FK, Figueiredo JL, et al. Impaired infarct healing in atherosclerotic mice with Ly-6C(hi) monocytosis. Journal of the American College of Cardiology. 2010;55(15): 1629-1638. [PubMed: 20378083]

55. Lee WW, Marinelli B, van der Laan AM, et al. PET/MRI of inflammation in myocardial infarction. Journal of the American College of Cardiology. 2012;59(2): 153-163. [PubMed: 22222080]

56. Rischpler C, Dirschinger RJ, Nekolla SG, et al. Prospective evaluation of 18F-fluorodeoxyglucose uptake in postischemic myocardium by simultaneous positron emission tomography/magnetic resonance imaging as a prognostic marker of functional outcome. Circulation. Cardiovascular Imaging. 2016;9(4):e004316. [PubMed: 27056601]

57. Alam SR, Shah AS, Richards J, et al. Ultrasmall superparamagnetic particles of iron oxide in patients with acute myocardial infarction: early clinical experience. Circulation. Cardiovascular Imaging. 2012;5(5):559-565. [PubMed: 22875883]

58. Stirrat CG, Alam SR, MacGillivray TJ, et al. Ferumoxytol-enhanced magnetic resonance imaging assessing inflammation after myocardial infarction. Heart. 2017; 103(19): 1528-1535. [PubMed: 28642288] 

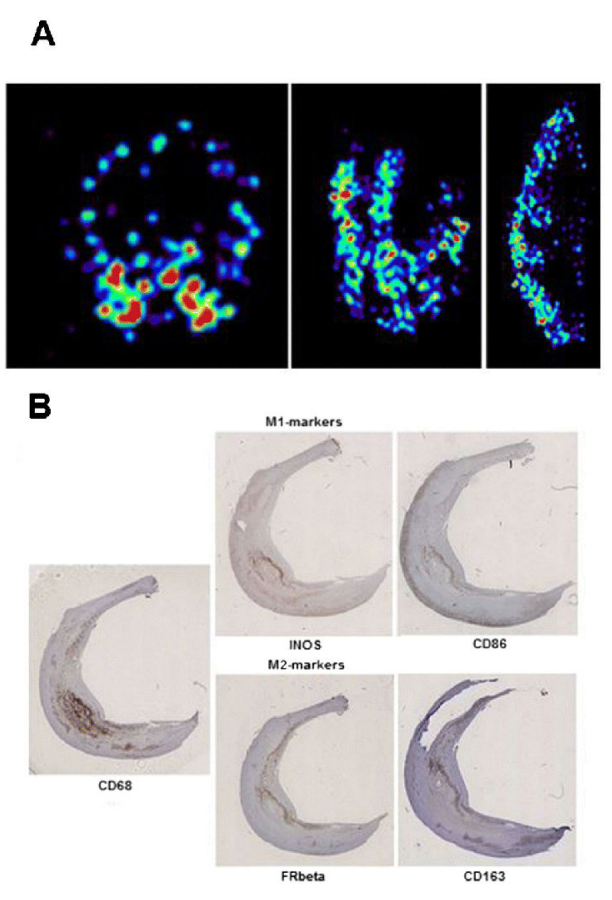
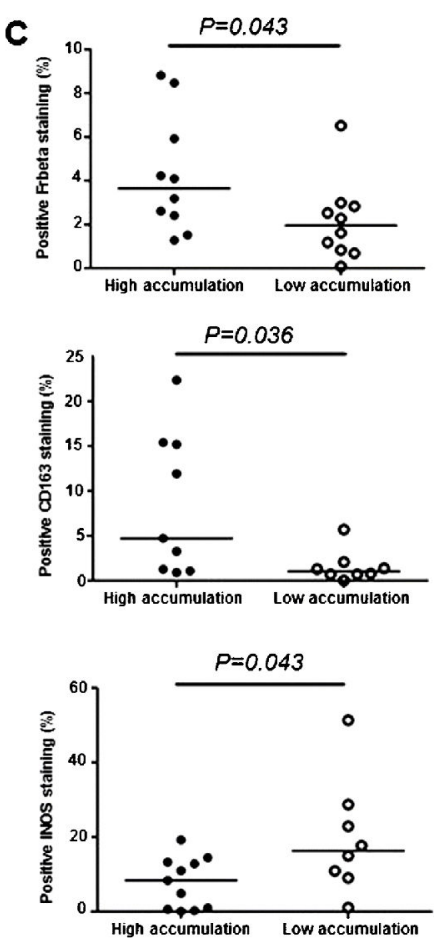

Figure 1: MicroSPECT images and histological analysis of ex vivo carotid endarterectomy specimens showing heterogeneous distribution of ${ }^{99 M}$ Tc-folate that corresponds to FR- $\beta$ staining.

A) Transverse (left), coronal (middle), and sagittal (right) sections of arterial specimens after incubation with ${ }^{99 \mathrm{M}} \mathrm{Tc}$-folate. The highest uptake is seen inside the arterial lumen on transverse section. B) Atherosclerotic plaques with the highest uptake of ${ }^{99 \mathrm{M}} \mathrm{Tc}$-folate showed the highest staining of FR- $\beta$ and CD163 (M1 markers) compared to INOS and CD86 (M2 markers). C) Comparison of the percent of cells with FR- $\beta$, Cd163, and INOS staining in slices with high and low accumulation of ${ }^{99 \mathrm{M}} \mathrm{Tc}$-folate. Adapted from Jager, et al. Journal of Nuclear Medicine, 2014, 55(12): 1945-1951. ${ }^{22}$ 

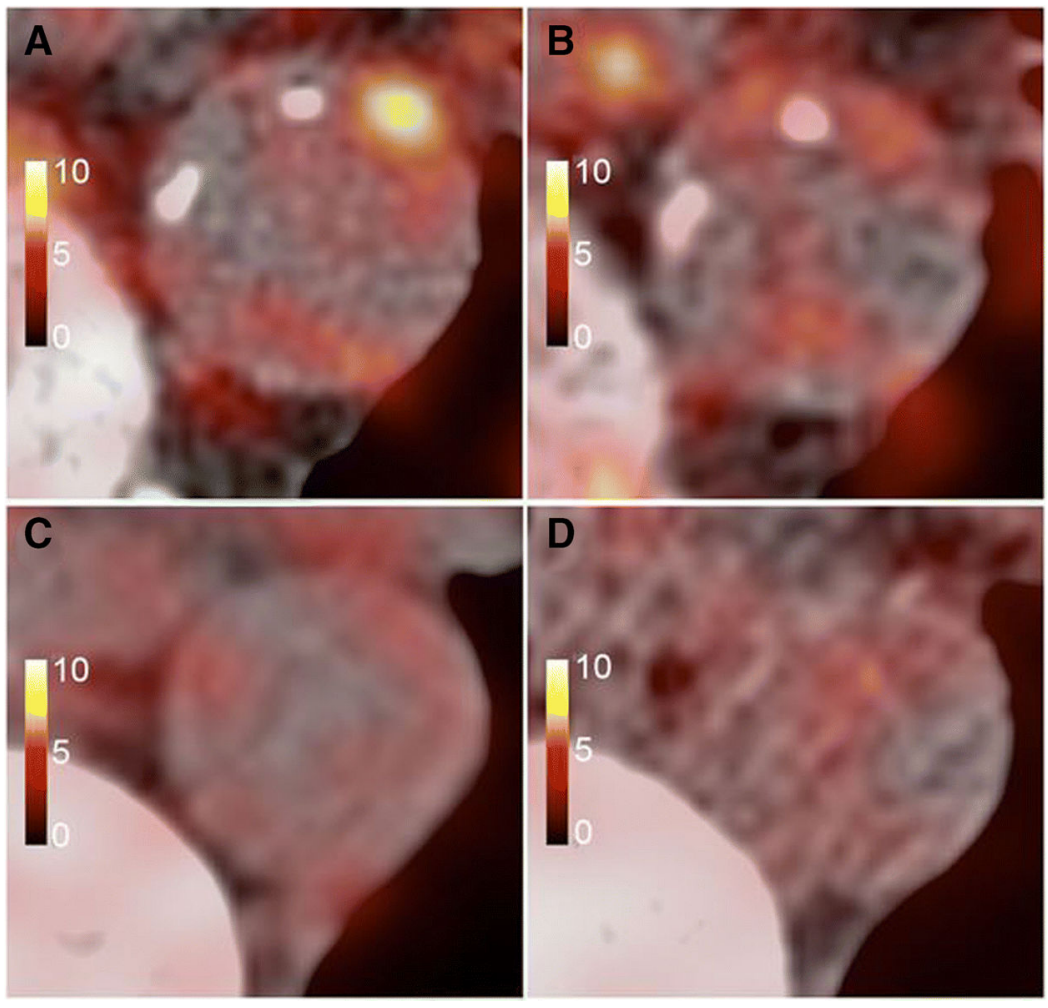

Figure 2: Comparison of PET/CT fusion images of ${ }^{64} \mathrm{Cu}$-DOTATATE (A and C) and ${ }^{68} \mathrm{Ga}$ DOTATOC (B and D).

Images A and B are depict respective tracer uptake in a 67 year old man with a Framingham risk score of 30. Note the high focal uptake of ${ }^{64} \mathrm{Cu}$-DOTATATE (A) compared to the blurred images of ${ }^{68} \mathrm{Ga}$-DOTATOC (B). Images $\mathrm{C}$ and $\mathrm{D}$ are taken from a 31 -year old woman with Framingham risk score of 2 . There is lower and more diffuse uptake of both tracers. Reprinted with permission from Malmberg C, et al. J Nucl Med, 2015;56(12): $1895-1900 .{ }^{45}$ 
Table 1:

Select list clinical imaging studies of IHD reported over the past 5 years

\begin{tabular}{|c|c|c|c|}
\hline \multicolumn{4}{|c|}{ Atherosclerosis } \\
\hline Author (year), n & Imaging Strategy & $\begin{array}{l}\text { Tissue, Risk factor or } \\
\text { Outcome Correlation }\end{array}$ & Findings \\
\hline Sadat, et al. ${ }^{48}$ (2013), n=10 & $\begin{array}{l}\text { Ultra-small SPION enhanced MRI } \\
\text { in patients with moderate carotid } \\
\text { disease }\end{array}$ & No & $\begin{array}{l}\text { Uptake in ultra-small SPIONs that did } \\
\text { not vary with serial imaging }\end{array}$ \\
\hline $\begin{array}{l}\text { Mojtahedi, et al. }{ }^{41} \text { (2015), } \\
n=44\end{array}$ & $\begin{array}{l}\text { (68) Ga-DOTATATE PET/CT } \\
\text { compared to CT with } \\
\text { neuroendocrine tumors }\end{array}$ & No & $\begin{array}{l}\text { Increase uptake in vulnerable or fibrotic } \\
\text { plaques compared to normal arteries }\end{array}$ \\
\hline $\begin{array}{l}\text { Pedersen, et al. }{ }^{44}(2015) \text {, } \\
n=10\end{array}$ & $\begin{array}{l}\text { (64) Cu-DOTATATE PET/MRI in } \\
\text { patients undergoing carotid } \\
\text { endarterectomy }\end{array}$ & Yes: gene expression & $\begin{array}{l}\text { Uptake higher in symptomatic compared } \\
\text { to asymptomatic arteries. Lesions with } \\
\text { higher uptake expressed genes } \\
\text { associated with plaque vulnerability. }\end{array}$ \\
\hline $\begin{array}{l}\text { Malmberg, et al. }{ }^{45} \text { (2015), } \\
n=60\end{array}$ & $\begin{array}{l}\text { (68) Ga-DOTATOC vs. (64)Cu- } \\
\text { DOTATATE PET/CT in patients } \\
\text { with neuroendocrine tumors }\end{array}$ & Yes: Framingham risk score & $\begin{array}{l}\text { (64) } \mathrm{Cu} \text {-DOTATATE PET/CT was } \\
\text { superior with higher uptake in vascular } \\
\text { lesions, which correlated with } \\
\text { Framingham risk score. }\end{array}$ \\
\hline $\begin{array}{l}\text { Tarkin, et al. }{ }^{42} \text { (2017), } \\
\mathrm{n}=42\end{array}$ & $\begin{array}{l}\text { (68) Ga-DOTATATE PET vs. } \\
\text { [(18)F]FDG PET imaging of } \\
\text { patients with unstable/stable carotid } \\
\text { or coronary diseaes. CT also } \\
\text { performed. }\end{array}$ & $\begin{array}{l}\text { Yes: gene expression, CT, } \\
\text { Framingham risk score }\end{array}$ & $\begin{array}{l}\text { (68) Ga-DOTATATE PET improved } \\
\text { image quality and had better power to } \\
\text { discriminate between high and low risk } \\
\text { plaque. }\end{array}$ \\
\hline Wan, et al. ${ }^{43}$ (2017), n=20 & $\begin{array}{l}\text { (68) Ga-DOTATATE PET/CT in } \\
\text { patients with recent TIA or stroke }\end{array}$ & Yes: histology & $\begin{array}{l}\text { No difference between disease and non- } \\
\text { diseased arteries. No evidence of } \\
\text { somatostatin receptor on macrophages }\end{array}$ \\
\hline \multicolumn{4}{|c|}{ Infarcted Myocardium } \\
\hline Author (year), n & Imaging Strategy & $\begin{array}{l}\text { Tissue, Risk factor } \\
\text { or Outcome } \\
\text { Correlation }\end{array}$ & Findings \\
\hline Alam, et al. ${ }^{57}$ (2012), n=16 & Ultra small SPIONs after STEMI & No & $\begin{array}{l}\text { Ultra-small SPIONs found in infarcted } \\
\text { myocardium that increased over } 48 \\
\text { hours. A similar but lower amount of } \\
\text { SPIONs found in the remote } \\
\text { myocardium. }\end{array}$ \\
\hline $\begin{array}{l}\text { Rischpler, et al. } .^{58} \text { (2016), } \\
n=49\end{array}$ & $\begin{array}{l}\text { (18) F-FDG PET/MRI with f/u MRI } \\
\text { in patients after STEMI }\end{array}$ & $\begin{array}{l}\text { Yes: MRI LVEF and infarct } \\
\text { size }\end{array}$ & $\begin{array}{l}\text { Mean SUV was associated with left } \\
\text { ventricular functional outcome } \\
\text { independent of infarct size }\end{array}$ \\
\hline Stirrat, et al. ${ }^{58}(2017), \mathrm{n}=31$ & Ultra small SPION enhanced MRI & Yes: Histology in 3 patients & $\begin{array}{l}\text { Ultra-small SPIONs found in remote } \\
\text { myocardium, which peaked within 2-3 } \\
\text { days and dissipated by week 2-post } \\
\text { myocardial infarction. }\end{array}$ \\
\hline
\end{tabular}

FDG: 2-deoxy-2-[ ${ }^{18}$ F]fluoro-D-glucose; LVEF; left ventricular function; MRI: magnetic resonance imaging; PET: positron emission tomography; SPION: superparamagnetic iron oxide nanoparticles; STEMI: ST elevation myocardial infarction; SUV: standardized uptake value 